\title{
Neovascular tissue in the intertrabecular spaces in eyes with neovascular glaucoma
}

\author{
Toshiaki Kubota, Akihiko Tawara, Yasuaki Hata, Ahmad Khalil, Hajime Inomata
}

\begin{abstract}
Aims/background-To investigate histological changes in the trabecular meshwork in eyes with neovascular glaucoma.

Methods-Light and electron microscopic studies were carried out on the trabecular meshwork of three enucleated eyes with neovascular glaucoma. The presence and distribution of factor VIII in the trabecular meshwork was assessed using the ABC method.

Results-Peripheral anterior synechiae covering the trabecular meshwork were detected in two eyes, which would explain the rise in intraocular pressure. In the third the angle was not completely closed by peripheral anterior synechiae. The spaces between the trabecular beams were lined by a single layer of vascular endothelium, and were filled with red blood cells in this patient. Factor VIII was positively stained in the endothelial cells, lining both these spaces and Schlemm's canal. A basal lamina and microfibrils were detected just beneath the newly formed vascular endothelial cells.
\end{abstract}

Conclusion-The neovascular tissue found in the trabecular spaces might be one of the factors responsible for intraocular pressure elevation in eyes with neovascular glaucoma.

(Br f Ophthalmol 1996;80:750-754)

Neovascular glaucoma is a severe potential complication after such ocular ischaemic diseases as diabetic retinopathy and central retinal vein occlusion. The mechanism of intraocular pressure elevation is considered to be caused by increased permeability of the newly formed vessels ${ }^{1}$ and angle closure by peripheral anterior synechiae. ${ }^{2}$ Although there have been several histological reports describing the trabecular meshwork in eyes with neovascular glaucoma, ${ }^{2-7}$ its ultrastructural changes have not yet been elucidated. In the present study, we investigated the ultrastructural changes of the trabecular meshwork to elucidate other possible reasons for the elevation of intraocular pressure (IOP) in eyes with neovascular glaucoma.

Toshiaki Kubota,

Department of

Ophthalmology, Faculty of

Medicine, Kyushu

University, 3-1-1 Maidashi,

Higashi-ku, Fukuoka 812,

Japan.

Accepted for publication 4 April 1996

\section{Materials and methods}

Three eyes with neovascular glaucoma which were all enucleated because of intolerable ocular pain were used in this study.
Patient 1

A 52-year-old man who had experienced visual disturbance since July 1992 presented with right ocular pain in May 1993. The right eye demonstrated no light sense and the intraocular pressure was $50 \mathrm{~mm} \mathrm{Hg}$. The clinical diagnosis was central retinal vein occlusion and secondary neovascular glaucoma in the right eye. Gonioscopic examination revealed that half of the angle was closed by peripheral anterior synechiae, while the other half was narrow but not completely closed. He had been treated with antiglaucomatous drugs without any operative intervention including cryotherapy. The right eye was enucleated on 10 February 1994.

\section{Patient 2}

A 40-year-old man who had had diabetes mellitus since he was 20 years old, underwent cataract operations in both eyes 8 years previously. He presented with visual disturbance in both eyes on 23 February 1994. Visual acuity was limited to hand movement in both eyes. The IOP was $13 \mathrm{~mm} \mathrm{Hg}$ in the right eye and $45 \mathrm{~mm} \mathrm{Hg}$ in the left. The clinical diagnosis was proliferative diabetic retinopathy and aphakia in both eyes, and secondary neovascular glaucoma in the left eye. Gonioscopic examination revealed secondary angle closure by peripheral anterior synechiae all around. His left eye was enucleated on 24 March 1994.

\section{Patient 3}

A 58-year-old man who had suffered an ocular injury in the right eye as a schoolchild, later developed ocular pain in the same eye. He consulted an ophthalmologist in August 1990 because of pain in his right eye. Visual acuity was limited to hand movement in that eye, and the IOP was $40 \mathrm{~mm} \mathrm{Hg}$. He had already undergone cryotherapy in the right eye. Gonioscopic examination revealed secondary angle closure by peripheral anterior synechiae all around. His eye was enucleated in June 1991 because of the unresolved pain.

Each globe was divided into two halves, one half was immediately fixed in a solution of $4 \%$ glutaraldehyde with $0.1 \mathrm{M}$ cacodylate buffer for 24 hours, dehydrated in graded ethanol, postfixed, and embedded in an epoxy resin. Semithin sections were cut with an ultramicrotome, stained with Azur II and examined with a light microscope. Ultrathin sections were stained with uranyl acetate and lead citrate, and then examined with an electron microscope (JEM-100CX). 
IMMUNOHISTOCHEMICAL PROCEDURE FOR VON WILLEBRAND FACTOR

The other halves of the eyes were fixed with $10 \%$ formaldehyde and then embedded in paraffin. For immunohistochemistry, the sections were deparaffinised with xylene, rehydrated with $0.01 \mathrm{M}$ phosphate buffered saline $(\mathrm{pH}$ 7.4) and pretreated with $0.1 \%$ trypsin in PBS for 30 minutes at $37^{\circ} \mathrm{C}$. After blocking with $1.5 \%$ skim milk for 1 hour, 800 times diluted rabbit anti-human von Willebrand factor antibody (Dakopatts, Denmark) was mounted, then incubated with biotinylated goat antirabbit IgG (Vector Laboratories, Inc, Burlingame, USA) for 30 minutes at room temperature. The sections were soaked in absolute methanol containing $0.3 \%$ hydrogen peroxidase for 30 minutes at room temperature to eliminate endogenous peroxidase activity and then were soaked in peroxidase labelled streptavidin (Vector Laboratories, Inc, Burlingame, USA) for 30 minutes at room temperature and rinsed as described above. Peroxidase activity was visualised by incubating the sections with 3-amino-9-ethyl carbazole (AEC, Dako, Tokyo, Japan). The sections were then mounted and examined with a light microscope.

Results

LIGHT MICROSCOPY

In one case (patient No 1), peripheral anterior synechiae were detected in only some areas of
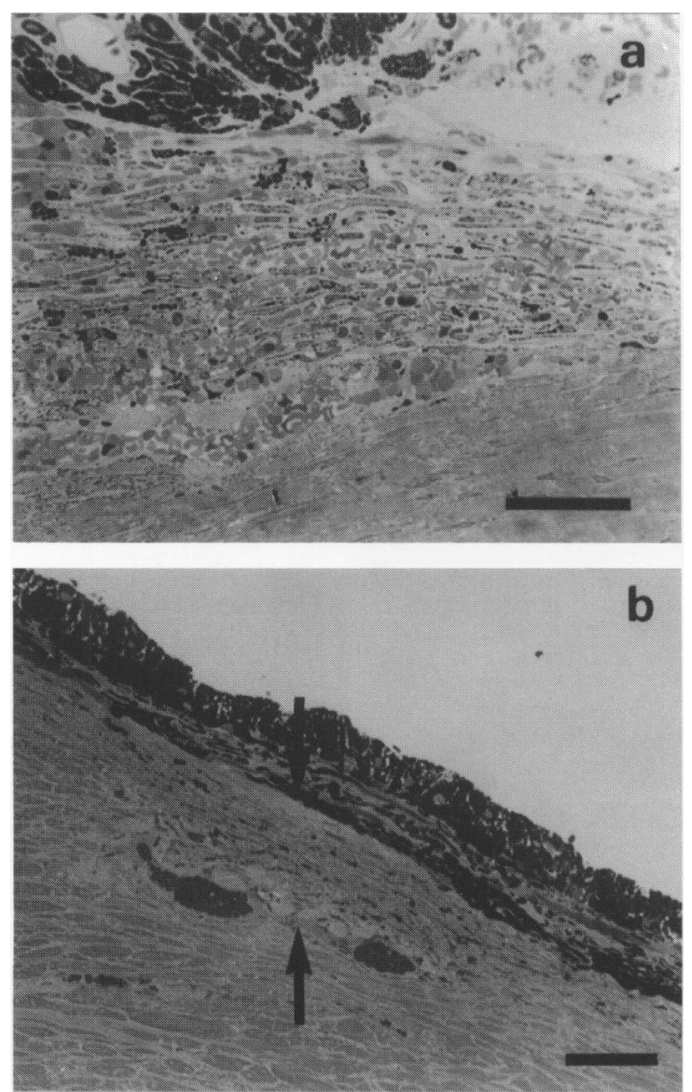

Figure 1 (a) (Patient 1) A light microscopic picture showing the trabecular meshwork not occluded by peripheral anterior synechiae. The spaces between the trabecular beams are filled with red blood cells. (b) (Patient 2) The trabecular meshwork was closed by a peripheral anterior synechia. The arrows indicate the area of the trabecular meshwork (Azur II staining). Bar $=50 \mu \mathrm{m}$.

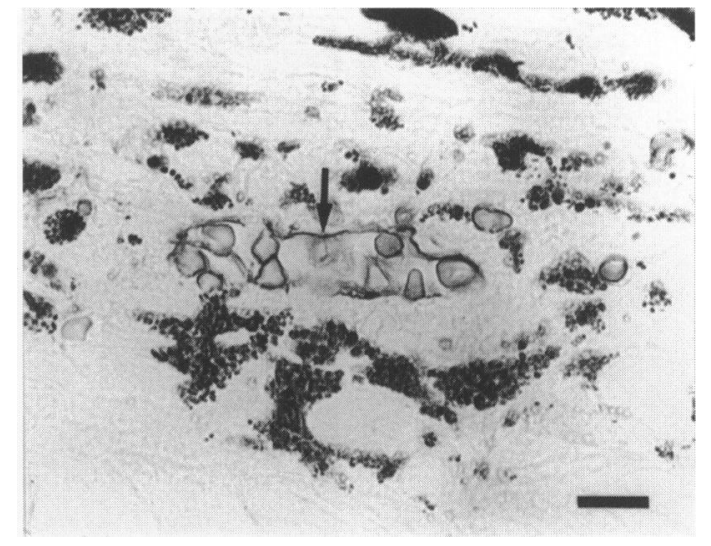

Figure 2 (Patient 1) Factor VIII is positively stained (arrow) in the endothelial cells covering the trabecular beams ( $A B C$ method). Bar $=10 \mu \mathrm{m}$.

the angle. A thin endothelial layer was detected overlying the trabecular meshwork in several sections. The spaces between the trabecular beams were lined by a single layer of cells and were filled with red blood cells and serum. In addition, Schlemm's canal was filled with red blood cells (Fig 1a). Factor VIII (von Willebrand) was positively stained in the cells lining the spaces between the trabecular beams and in the inner and outer walls of Schlemm's canal (Fig 2).

In the other two cases (patients 2 and 3), fibrovascular connective tissue adhered to the peripheral cornea and iris creating peripheral anterior synechiae. The trabecular meshwork showed a considerable degree of pigmentation. The trabecular beams were adherent together in many areas with cellular components between them. Some of these cells contained many pigment granules. Capillary-like structures filled with red blood cells were observed in the trabecular meshwork. Schlemm's canal was filled with red blood cells (Fig 1b).

\section{ELECTRON MICROSCOPY}

In patient No 1 , several trabecular beams were adherent together and most trabecular cells were degenerated in many areas. The spaces between the trabecular beams were lined with a single layer of endothelial cells. The trabecular cells were reduced in number. The remaining trabecular cells contained many pigment granules. Trabecular beams contained collagen fibrils, elastin-like fibres, and long spacing collagen fibrils. A homogeneous linear basal lamina was observed to be aligned with the basal surface of the trabecular cells (Fig 3). The layer of endothelial cells had junctional modifications and fenestrations. The intertrabecular spaces were filled with red blood cells and serum. The endothelial cells contained no pigment granules. The extracellular matrix associated with the endothelial cells consisted virtually of a basal lamina and microfibrils, which were observed just beneath the endothelial cells. In addition, the basal lamina was thin and appeared to be immature (Figs 3 and 4).

In patients 2 and 3, trabecular beams adhered together and most trabecular cells were degenerated and reduced in number. The remaining trabecular cells contained many pigment granules. In parts of the trabecular mesh- 

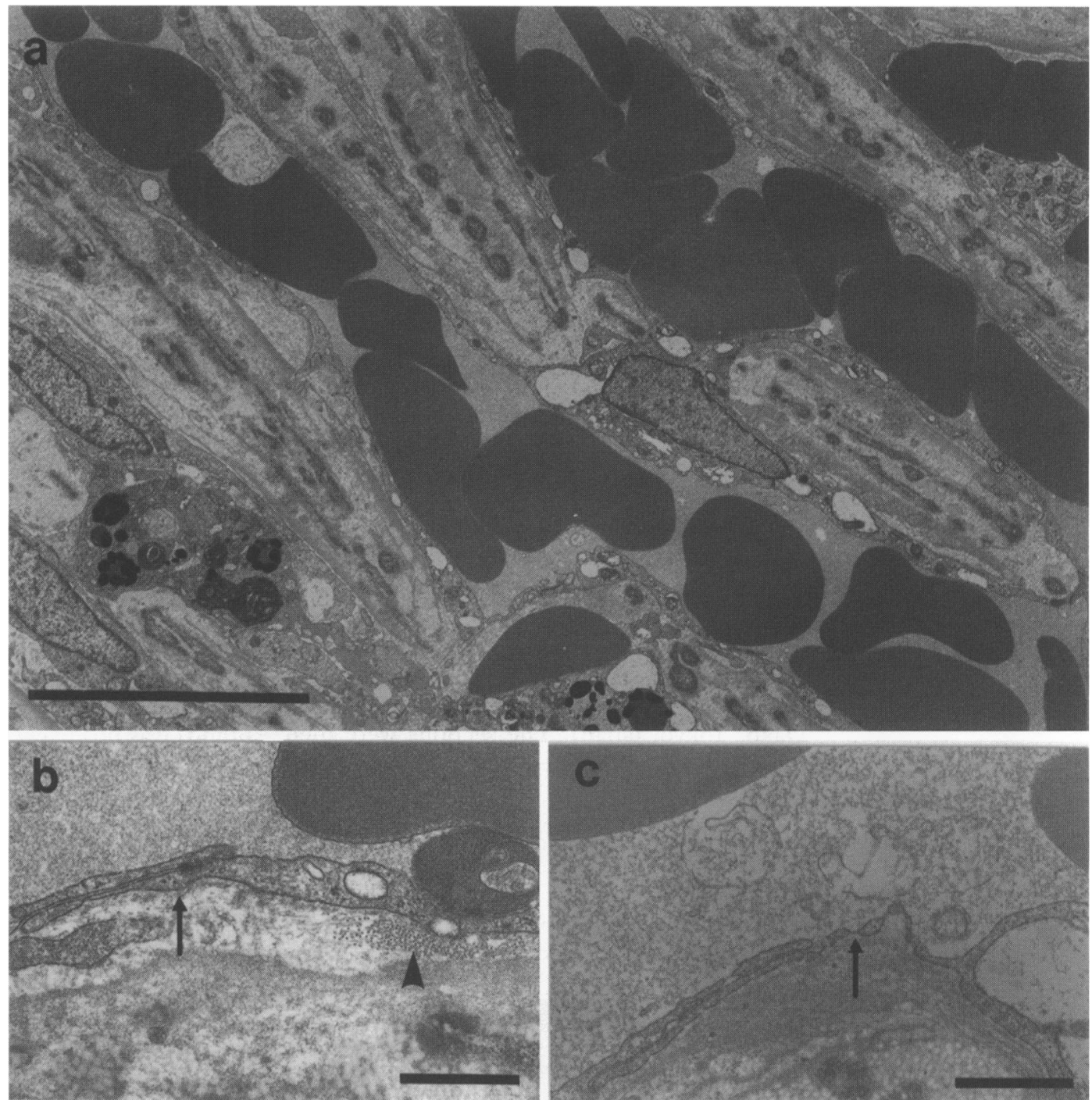

Figure 3 (Patient 1) Electron micrographs of the trabecular meshwork. (a) The intertrabecular spaces lined by the vascular endothelial cells are filled with red blood cells and plasma. The remaining trabecular cells contain pigment granules. Bar=10 $\mu \mathrm{m}$. (b) Microfibrils (arrowhead) and immature basal lamina (arrow) are found just beneath the vascular endothelial cells. Bar=1 $\mu \mathrm{m}$. (c) The endothelial cells lining the trabecular beams have fenestrae (arrow). Bar=1 $\mu \mathrm{m}$.

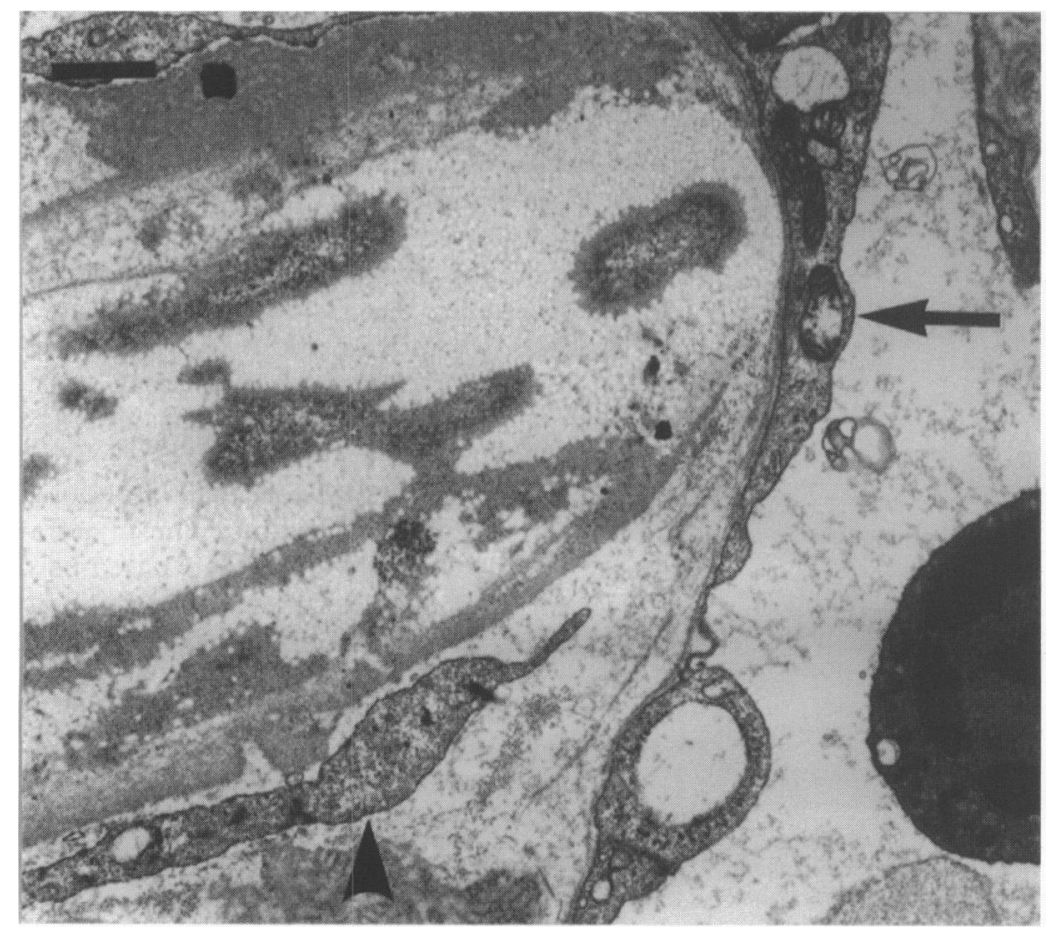

Figure 4 (Patient 1) Electron micrograph showing the vascular endothelium (arrow) with fenestrations and trabecular beam covered by degenerated trabecular cells (arrowhead). Bar=1 $\mu \mathrm{m}$. work, endothelial cells formed capillary-like structures. It was possible to identify cells resembling pericytes surrounding the endothelial cells. The cytoplasm of the pericytes was rich in ribosomes, and had intracytoplasmic microfilaments (Fig 5).

\section{Discussion}

In patients 2 and 3 , the IOP elevation can be explained by the presence of peripheral anterior synechiae and extension of Descemet's membrane to cover the trabecular meshwork. In patient No 1 , however, the angle was not completely closed by peripheral anterior synechiae. With light microscopy in this patient, the intertrabecular spaces appeared as if blood components had filled them. Electron microscopy, however, demonstrated that the endothelial cells lining the trabecular beams had fenestrations with junctional modifications between the cells. These cells were positively stained for factor VIII with pericytes occasionally adjacent to them. Factor VIII was reported to be positively stained in the endothelium of Schlemm's canal, as well as blood vessels of other parts of the eye, but not in trabecular cells. ${ }^{8}$ Degenerated trabecular cells containing 
many pigment granules were found in the trabecular tissue. We therefore concluded that the cells lining the trabecular beams were vascular endothelial cells of neovascular origin.

A histopathological staging of eyes with diabetic neovascular glaucoma, according to the formation and extension of fibrovascular tissue in the anterior chamber angle and on the iris surface, has divided the condition into four stages. ${ }^{3}$ Stage 1 , fibrovascular tissue occurs in the trabecular meshwork: although the angle is open, neovascular glaucoma develops; stage 2, fibrovascular tissue extends from the trabeculum into the anterior chamber: peripheral anterior synechiae develop because of shrinkage of fibrovascular tissue within the angle; stage 3, fibrovascular tissue spreads on the anterior surface of the iris; and stage 4 , a single layer of endothelial cells develops on the surface of the fibrovascular membrane overlying the iris. According to this classification, patient No 1 in our study would be classified into stage 2 , while the other two cases would be stage 4 . The ultrastructure of the trabecular meshwork of neovascular glaucoma in stage 2 has not yet been clarified. The present study showed that trabecular cells might degenerate and/or disappear in neovascular glaucoma. A single layer of the neovascular endothelial cells
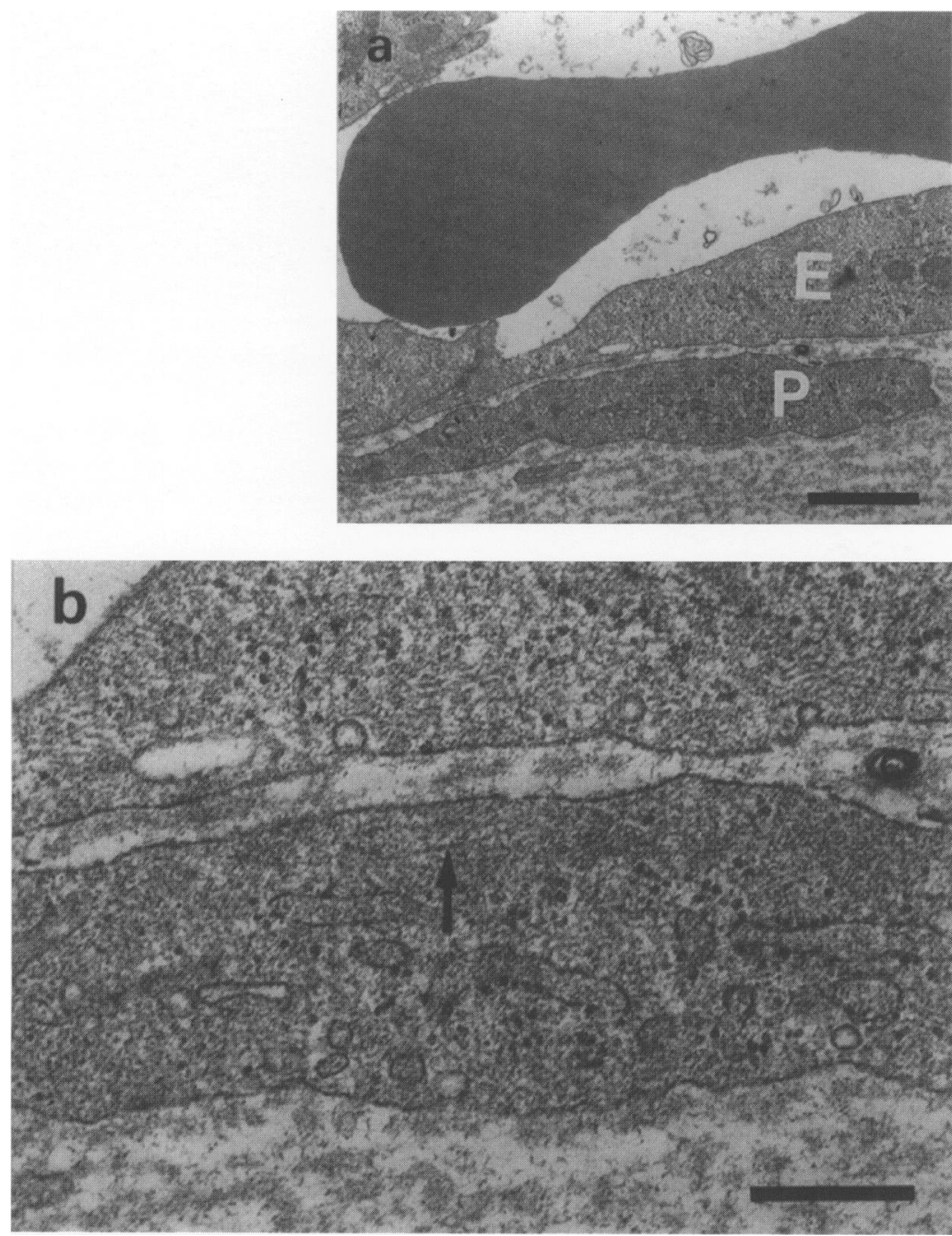

Figure 5 (Patient 2) (a) An electron micrograph of a pericyte sheathing an endothelial cell of the newly formed vessel. E, endothelial cell; P, pericyte. Bar $=1$ um. (b) Higher magnification of the pericyte. The cytoplasm of the pericyte is rich in ribosomes, and has intracytoplasmic microfilaments (arrow). Bar $=0.5 \mu \mathrm{m}$. lines the trabecular beams. It is reasonable to consider that the newly formed vessels impede the aqueous outflow through the trabecular meshwork. Thus, the neovascular tissue that occupied the intertrabecular spaces may be one of the factors responsible for the IOP elevation in eyes with neovascular glaucoma.

The fine structure of the newly formed vessels has been investigated in other ocular tissues ${ }^{910}$ and in vitro. ${ }^{11}$ The endothelial cells of newly formed vessels in subretinal neovascularisation have a basal lamina and form capillary structures with junctional modifications and pericytes adjacent to them. ${ }^{910}$ The pericytes have microfilaments preferentially located along the plasma membrane facing the endothelium. ${ }^{12}$ The close relation between neovascularisation and pericytes has been reported previously. ${ }^{13}$ Some authors ${ }^{14-16} \mathrm{de}-$ scribed that, in a coculture system of pericytes and endothelial cells, pericytes suppressed both endothelial proliferation and migration. They considered that the loss of pericytes may thus promote neovascularisation. In the present study, pericytes were found adjacent to vascular endothelial cells lining the trabecular beams. This finding also supports the observation that the endothelial cells lining trabecular beams in eyes with neovascular glaucoma are newly formed ones.

Normally, trabecular cells covering the trabecular beams are resting on a basal lamina. ${ }^{17}$ In this study, microfibrils and basal lamina were observed between the vascular endothelial cells and the trabecular cells. These structures are non-existent in the normal trabecular meshwork, and are considered to be produced in pathological conditions.

The presence of newly formed vessels in the trabecular meshwork was reported in the herpes simplex cases using immunohistochemistry and electron microscopy. ${ }^{7}$ The newly formed vessels were observed in some intertrabecular spaces in trabeculitis. Although their findings seemed very similar to the present ones, the intertrabecular spaces were completely occupied with the newly formed vessels in the present patient No 1. Using factor VIII immunohistochemical analysis and electron microscopy, we could not determine the origin of these endothelial cells at this stage. Further studies on eyes with earlier stages might help to resolve this problem. This work was supported by the grants from the Ministry of research \# 06771507 and grant in aid for joint research on research \# 06771507 and grant in aid for joint
pathogenesis of exfoliation syndrome \# 06044265).

1 Inomata $\mathrm{H}$, Iwasaki $\mathrm{M}$. Neovascular glaucoma. fpn $f$ Clin Ophthalmol 1991;45:100-1.

2 Gartner S, Henkind P. Neovascularization of the iris (rubeosis iridis). Surv Ophthalmol 1978;22:291-312.

3 Nomura T, Furukawa $\mathrm{H}$, Kurimoto S. Development and classification of neovascular glaucoma in diabetic eye disease: histopathological study. Acta Ophthalmol Soc $\mathfrak{f p}_{p n}$ 1976;86:166-75.

4 Gartner S, Taffet S, Friedman AH. The association of rubeosis iridis with endothelialization of the anterior chamber: report of a clinical case with histopathological review of 16 additional cases. Br f Ophthalmol 1977;61: 267-71.

5 Futa R, Shimizu T, Yoshizumi M. Electron microscopic study of the angle of the anterior chamber and the iris in diabetic neovascular glaucoma. Folia Ophthalmol fpn 1981 32:1095-106. 
6 Hamanaka T, Ujiie K, Kimura Y, Nitta A. Morphology and mode of progression in incipient chamber angle neovascularization. Folia Ophthalmol fpn 1981;32: 2526-40.

7 Lee WR. The pathology of the outflow system in primary and secondary glaucoma. Eye 1995;9:1-23.

8 Hamanaka T, Bill A, Ichinohasama R, Ishida T. Aspects of the development of Schlemm's canal. Exp Eye Res 1992;55: 479-88.

9 Miller H, Miller B, Ryan SJ. Newly formed subretina vessels. Fine structure and fluorescein leakage. Invest $O p h$ thalmol Vis Sci 1986;27:204-13.

10 Ishibashi T, Miller H, Orr G, Sorgente N, Ryan SJ. Morphological observations on experimental subretina neovascularization in the monkey. Invest Ophthalmol Vis Sci 1987;28:1116-30.

11 Murata T, Ishibashi T, Inomata H, Sueishi K. Media conditioned by coculture of pericytes and endothelial cells under a hypoxic state stimulate in vitro angiogenesis. Ophthalmic Res 1994;26:23-31.
12 Courtoy PJ, Boyles J. Fibronectin in the microvasculature: localization in the pericyte-endothelial interstitium. $f$ Ultrastruct Res 1983;83:258-73.

13 Ishibashi T, Inomata H, Sakamoto T, Ryan SJ. Pericytes of newly formed vessels in experimental subretinal neovascunewly formed vessels in experimental subretinal
larization. Arch Ophthalmol 1995;113:227-31.

14 Orlidge A, D'Amore PA. Inhibition of capillary endothelial cell growth by pericytes and smooth muscle cells. $\mathcal{F}$ Cell Biol 1987;105:1455-62.

15 Antonelli-Orlidge A, Saunders KB, Smith SR, D'Amore PA. An activated form of transforming growth factor $\beta$ is produced by cocultures of endothelial cells and pericytes. Proc Natl Acad Sci 1989;86:4544-8.

16 Sato Y, Rifkin DB. Inhibition of endothelial cell movement by pericytes and smooth muscle cells: activation of a latent transforming growth factor- $\beta 1$-like molecule by plasmin transforming growth factor- $\beta 1$-like molecule

17 Rohen JW. Anatomy of the aqueous outflow channels. In: Cairns JE, ed. Glaucoma. London: Grune \& Stratton, 1986:277-96. 